\title{
Antibiotic resistance in ophthalmology
}

\author{
Dominika Nowakowska $\odot^{1}$, Karolina Krix-Jachym², Marek Rękas², Edward Wylęgałaª , \\ Robert Rejdak (1) \\ 'Department of General and Pediatric Ophthalmology, Medical University of Lublin, Lublin, Poland \\ ${ }^{2}$ Department of Ophthalmology, Military Institute of Medicine, Warsaw, Poland \\ ${ }^{3}$ Clinical Department of Ophthalmology Medical University of Silesia, Katowice, Poland
}

\begin{abstract}
Antibiotic resistance is a very serious medical problem. In ophthalmology, it is still unnoticed and underestimated. Although antibiotic drops appear to be harmless, their inappropriate use may increase resistance in some bacteria. Many recommendations emphasize the importance of the correct use of antibiotics - according to the guidelines, antibiotic therapy should be intensive and short-term. Rational administration of antimicrobial drugs is essential when eye-threatening severe conditions such as endophthalmitis need to be prevented or treated.
\end{abstract}

KEY WORDS: antibiotic resistance; fluoroquinolones; cataract; endophthalmitis

Ophthalmol J 2021; Vol. 6, 117-123

\section{INTRODUCTION}

According to the definition of World Health Organization (WHO) experts, antibiotic resistance is the ability of a microorganism to maintain vital functions, including reproduction, despite exposure to a drug that was once effective against this microorganism [1]. Resistance is the property of the microorganism and not of the infected organism.

The causes of antibiotic resistance include:

- overprescribing of antibiotics;

- patient non-adherence to antibiotic therapy;

- overuse of antibiotics in agriculture (animal breeding);

- poor infection control in hospitals and clinics;

- insufficient level of hygiene and sanitary behavior;

- low availability of rapid laboratory tests confirming bacterial infections.

Antibiotic resistance develops naturally, mainly through gene mutations $[2,3]$, but the contemporary overuse of antibiotics increases this problem
[4]. Targeted metagenomic analysis of ancient DNA from permafrost deposits in the Bering region, dating back 30,000 years, revealed a very diverse set of genes encoding resistance to beta-lactam, tetracycline, and glycopeptide antibiotics [2]. These results clearly indicate that antibiotic resistance is a natural phenomenon that precedes the contemporary pressure resulting from the use of this group of drugs.

There are four well-known mechanisms for the development of antimicrobial resistance:

- enzymatic inactivation;

- modification of the drug target;

- active elimination (or efflux) of drugs from the microbial cell;

- impaired cell membrane permeability.

The mechanism of enzymatic inactivation is that bacteria synthesize enzymes that inactivate beta-lactam antibiotics. These enzymes include: plasmid penicillinases, extended-spectrum beta-lactamase, metallo-beta-lactamase, and chromosomal cephalosporinases [5]. Modification of the drug bind- 
ing point occurs through a conformational change in the large (50S) bacterial ribosome subunit. This is how resistance to macrolides (e.g., azithromycin) develops [6]. Atypical penicillin-binding proteins in staphylococci lead to the formation of methicillin-resistant Staphylococcus aureus (MRSA) strains [5]. Reduction of the cell membrane permeability [5] is the mechanism by which resistance to aminoglycosides in Pseudomonas aeruginosa (i.e., tobramycin) develops and resistance to thiazoles in Candida fungi. Antibiotic resistance can be produced by actively removing the drug from the cell (efflux). Pseudomonas aeruginosa can thus actively eliminate carbapenems and fluoroquinolones (ciprofloxacin, ofloxacin, and others) [5].

Antimicrobial resistance is a significant contributor to deaths each year, with incidence expected to increase more than 10-fold by 2050 [7]. According to $\mathrm{WHO}$ experts, addressing the issue of antibiotic resistance is essential to improving treatment for patients suffering from infections worldwide. Coordinated action on a global scale is needed to reduce the development and spread of resistance [1]. The 2014 WHO report on antibiotic resistance states: This serious threat is no longer a prediction for the future. It is happening right now in every region of the world and has the potential to affect anyone, of any age, in any country. Antibiotic resistance - when bacteria change, so antibiotics no longer work in people who need them to treat infections - is now a significant threat to public health [1]. Data published by the European Center for Disease Prevention and Control (ECDC) indicate that antibiotic-resistant bacteria were the causes of 671,689 infections in the European Union (EU) and the European Economic Area (EEA), resulting in 33,110 deaths in 2015 [3]. Most of these infections were acquired in healthcare facilities. Deaths from antibiotic-resistant microbial infections occurred mostly in infants and those 65 years of age or older. Moreover, since 2007 an upward trend of this phenomenon has been observed.

Off-label use of drugs plays a significant role in the development of antibiotic resistance. Both the patients and the doctors are to blame. Prescribing antibiotics at lower dosages can increase drug resistance by developing more mutations to support bacterial growth. This applies to drugs (including topically administered fluoroquinolones) whose pharmacokinetics is dose-dependent, so therapy's effectiveness depends on the drug's concentration in the blood or other fluids and the minimum inhibitory concentration (MIC). For example, it is known that sub-inhibitory concentrations caused a genetic mutation in bacteria such as Pseudomonas aeruginosa and Bacteroides fragilis [8].

\section{PHARMACOTHERAPY IN CATARACT SURGERY Antibiotic therapy}

Antibiotic therapy is obligatory for preoperative inflammatory conditions such as eyelid margin inflammation, conjunctivitis, hordeolum, chronic tear sac inflammation, second-eye infections, and chalazion [9]. However, cataract surgery is not considered an invasive procedure that causes transient bacteremia, and therefore general antibiotic prophylaxis is not necessary $[4,9]$. If nevertheless, the surgeon is considering oral antibiotic preoperative prophylaxis, he or she should consult the patient's GP. The effectiveness of local antibiotic prophylaxis in preventing endophthalmitis has not been confirmed in reliable scientific studies [10]. Some guidelines allow the use of fluoroquinolones before cataract surgery [11]. Drug administration regimens range from the first-hour post to 3 days prior to surgery.

Despite surgeons' best efforts, $7-35 \%$ of cataract operations involve the inoculation of bacteria into the anterior chamber. Despite this, the percentage of endophthalmites after cataract surgery is low because the anterior chamber has the ability to self-cleanse from the introduced bacteria [13]. There are many methods of administering antibiotics perioperatively. Some surgeons add cefuroxime to the irrigation fluid or perform a subconjunctival injection after surgery, which can achieve a bactericidal concentration in the anterior chamber for 1-2 hours. Still, neither of these methods has been proven effective in preventing postoperative endophthalmitis. Lindstrom et al. introduced the concept of "dropless cataract surgery" [12]. This technique involves a single intravitreal injection of fluoroquinolone in combination with a glucocorticosteroid. A single-use product is available in the United States: a premixed, combined, injectable formulation containing triamcinolone $(15 \mathrm{mg} / \mathrm{mL})$ and moxifloxacin $(1 \mathrm{mg} / \mathrm{mL})$. The surgeon injects $0.2 \mathrm{mg}$ of the preservative-free suspension into the vitreous chamber. So far, the results of using this preparation are very promising. This method results in an essential change in the doctor-patient rela- 
tionship. When the surgeon administers the drug directly, he does not have to rely on the patient's adherence. At the same time, this strategy provides a greater likelihood of obtaining the correct dose and greater certainty as to the final result of the treatment. This drug is not available in Poland. An undisputed method of endophthalmitis prevention is the intracameral administration of cefuroxime $(1 \mathrm{mg} / 0.1 \mathrm{~mL})$ at the end of cataract surgery. A statistically significant decrease in the incidence of endophthalmitis after cataract surgery was observed. These results were confirmed in a large, randomized study, on the basis of which experts from the European Society of Cataract and Refractive Surgeons (ESCRS) developed guidelines for the prevention and treatment of endophthalmitis after cataract surgery [4]. These guidelines also recommend the use of antibiotic drops after this procedure. Although the study conducted by the ESCRS, Endophthalmitis Study Group itself, did not confirm the effectiveness of topical antibiotics after surgery, in cases of uncertainty about the tightness of surgical incision closure, their use was justified. Fluoroquinolones were considered to be the most preferred. It was emphasized that these drugs should be used intensively and briefly, without gradual discontinuation, which may contribute to the development of antibiotic resistance [4].

Anti-inflammatory treatment includes local steroid administration for 21 days in decreasing doses to minimize the inflammatory reaction $[9,14]$. Steroid drugs relieve pain, prevent fibrin formation, prevent the development of adhesions and reduce photophobia. It has been proven that steroid drop therapy after cataract surgery reduces the risk of secondary cataract development [14]. Drops with steroids should be discontinued gradually after prolonged use to prevent recurrence of the inflammatory reaction and adrenal crisis (reducing the dose of the drug gives the adrenal glands time to return to standard secretion patterns) [15]. In all people at increased risk of developing macular cystic edema (i.e., with diabetes, after uveitis, after complicated cataract surgery, patients using prostaglandins for glaucoma during the perioperative period, etc.), it is recommended to use an additional non-steroidal anti-inflammatory drug (NSAID), preferably in combination with a steroid according to the SPC [16]. In patients at increased perioperative risk (e.g., with recurrent uveitis), systemic administration of corticosteroids should be considered using the approved dosing regimen [9].

\section{The rationale for the use of fluoroquinolones}

The main factors to consider when choosing the optimal antibiotic therapy are:

- etiopathogenesis of the disease and patterns of resistance;

- the clinical situation in which the disease occurs;

- disease severity;

- natural and acquired antibiotic resistance of pathogens;

- clinical pharmacology of the antibiotic;

- results of clinical trials with antibiotics.

The 2013 ESCRS guidelines for the prevention and treatment of endophthalmitis after cataract surgery [4] provide an indication that justifies the use of topical antibiotics perioperatively. However, their efficacy has not been proven in prospective scientific studies. This applies to cases where there is uncertainty about the tightness of surgical incisions, when complications occur, or when the patient is exposed to a nosocomial infection or has symptoms of an upper respiratory tract infection. Fluoroquinolones are considered the drugs of choice due to their broad spectrum of action, ability to penetrate the corneal epithelium, and general availability.

Fluoroquinolones belong to quinolones, a class of synthetic antibiotics. Quinolones are divided into four generations (Tab. 1).

The bactericidal activity of quinolones is based on blocking bacterial enzymes (topoisomerase II, a DNA gyrase, and topoisomerase IV) necessary for the further multiplication and development of bacteria. Quinolones are active against both DNA gyrase and topoisomerase IV, but their relative activities against these enzymes differ. Hooper et al. [17] initially stated that the main target of quinolone activity was DNA gyrase, and topoisomerase IV seemed to be a secondary target. In contrast, later studies on $S$. aureus found that the primary target of quinolones in this species was topoisomerase IV, in contrast to earlier findings in E. coli studies. Older-generation quinolones differ in their relative activities towards DNA gyrase and topoisomerase IV in a bacterial cell. In gram-positive bacteria, they are more potent against gyrase than against topoisomerase IV. In Gram-negative bacteria, the effect on topoisomerase IV is more significant than on gyrase in many Gram-positive bacteria.

In contrast, newer quinolones, including fluoroquinolones and polyfluoroquinolones, appear to have more balanced activity against the two enzyme targets. The findings so far also highlight different levels of quinolone resistance depending on whether 


\begin{tabular}{|c|c|c|c|}
\hline $\begin{array}{l}\text { Quinolones } \\
1^{\text {st }} \text { generation }\end{array}$ & $\begin{array}{l}\text { Fluoroquinolones } \\
2^{\text {nd }} \text { generation }\end{array}$ & $\begin{array}{l}\text { Polyfluoroquinolones } \\
3^{\text {rd }} \text { generation }\end{array}$ & $\begin{array}{l}\text { Naphthyridinequinolones } \\
4^{\text {th }} \text { generation } * * *\end{array}$ \\
\hline & & $\begin{array}{l}\text { Extended spectrum of action, increased } \\
\text { potency and an extended half-life }\end{array}$ & $\begin{array}{l}\text { Spectrum of action as the previous } \\
\text { generation, increased activity against } \\
\text { resistant Gram-positive bacteria and } \\
\text { better penetration into the ocular tissues } \\
\text { (conjunctiva, cornea) }\end{array}$ \\
\hline Nalidixic acid & Pefloxacin & Grepafloxacin & Moxifloxacin \\
\hline Pipemidic acid* & Ciprofloxacin** & Levofloxacin & Gemifloxacin \\
\hline \multirow[t]{6}{*}{ Cinoxacin } & Norfloxacin & Pazufloxacin & Clinafloxacin \\
\hline & Ofloxacin & Sparfloxacin*** & Trovafloxacin*** \\
\hline & Fenoxacin & Tosufloxacin & Lomefloxacin \\
\hline & Fleroxacin & & Gatifloxacin \\
\hline & Lomefloxacin & & Des 6-chinolon \\
\hline & Temafloxacin & & \\
\hline
\end{tabular}

*also has moderate activity against Pseudomonas aeruginosa; ${ }^{* *}$ has the highest activity against Pseudomonas aeruginosa; ${ }^{* * *}$ withdrawn (due to toxicity); ****has activity also against penicillin-resistant Streptococcus pneumoniae, and also against anaerobes

the primary or secondary target is altered or both. Mutations in the primary target precede those in the secondary target in gradual selection for resistance, while mutations in both targets increase the level of resistance [17].

Polyfluoroquinolones (e.g., levofloxacin) are characterized by an extended spectrum of action, increased potency, and a prolonged half-life compared to the older generation of fluoroquinolones (e.g., ofloxacin) $[18,19]$. Table 2 shows the range of antibacterial activity of polyfluoroquinolone levofloxacin.

Polyfluoroquinolones are highly effective in the treatment of bacterial infections of the anterior segment of the eye. In a study involving 208 patients

\begin{tabular}{|c|c|}
\hline Gram-positive bacteria & Gram-negative bacteria \\
\hline Staphylococcus aureus* & Pseudomonas aeruginosa* \\
\hline Staphylococcus epidermidis* & Moraxella catarrhalis \\
\hline Streptococcus pneumoniae* & Haemophilus influnzae* \\
\hline \multirow[t]{6}{*}{ Streptococcus pyogenes* } & $\begin{array}{c}\text { Enterobacteriacae family*: } \\
\text { Neiseria meningitidis } \\
\text { Gonorrhoeae }\end{array}$ \\
\hline & Mycobacterium tuberculosis* \\
\hline & Chlamydia \\
\hline & Legionella \\
\hline & Mycoplasma \\
\hline & Ureoplasma \\
\hline
\end{tabular}

*pathogens that are also the physiological flora of the conjunctiva and potential etiological factors of endophthalmitis suffering from bacterial conjunctivitis, Schwab et al. assessed levofloxacin's efficacy and safety profile [20]. Levofloxacin has been shown to be more effective against $H$. influenzae and $S$. pneumonia than ofloxacin [20]. The degree of microbial eradication was statistically significantly higher in patients receiving levofloxacin compared with the control group receiving ofloxacin. In addition, subjective symptoms such as photophobia resolved much faster in levofloxacin-treated patients than in ofloxacin-treated patients ( $94 \%$ vs. $73 \%, \mathrm{p}=0.006$ ). Both drugs were well tolerated [20].

Another advantage of this group of drugs is its pharmacokinetics. Topical levofloxacin has been shown to penetrate the tissues of the eye readily. This results from the lipophilicity and good solubility of this drug [21]. Sakamoto et al. noted that levofloxacin is ten times more soluble than ofloxacin and up to 400 times more soluble than ciprofloxacin [22]. In addition, the above properties allow levofloxacin to achieve a concentration in the tear film that exceeds the MIC90 (minimum concentration of antibiotic in the culture at which $90 \%$ of the selected bacterial strain has no evidence of reproduction). For fluoroquinolones used against most ophthalmic pathogens, MIC90 is $\leq 2 \mu \mathrm{g} / \mathrm{mL}$ [23].

Raizman et al. [24] showed that the administration of levofloxacin is beneficial in non-adherent patients. The study involved healthy volunteers who received one drop of $0.5 \%$ levofloxacin into the conjunctival sac. The concentration of the drug in tears peaked 15 minutes after administration $(221.06 \mu \mathrm{g} / \mathrm{mL})$, then decreased after 6 hours after 
administration, reaching the value of $6.57 \mu \mathrm{g} / \mathrm{mL}$. Interestingly, 24 hours after the administration of levofloxacin, $33 \%$ of the examined patients had concentrations of levofloxacin exceeding $2.0 \mu \mathrm{g} / \mathrm{mL}$. The authors concluded that $0.5 \%$ levofloxacin meets and exceeds the MIC90 value and therefore has a prolonged effect which facilitates dosing as no additional overnight administration is required.

The newest-generation fluoroquinolones (e.g., moxifloxacin) have the same spectrum of action as the previous generation but with increased activity against resistant gram-positive bacteria and better penetration into the ocular tissues (conjunctiva, cornea) [25]. However, their use as first-line drugs is not recommended to not produce antibiotic resistance [25].

\section{DOCTOR-PATIENT COOPERATION AND PATIENT ADHERENCE TO TREATMENT}

Many studies are examining the factors that adversely affect patient adherence to treatment. Regarding ophthalmic drops, difficulties are often caused by the instillation of the drug into the conjunctival sac, keeping the dropper clean, using the drug regularly and for a designated time - not longer and not shorter than required by the therapy $[26,27]$. Problems arise when a patient has to take multiple medications. Patients mistake containers for drops or fail to buy one of the drugs, for example, for economic reasons. This lack of cooperation translates into a therapeutic effect, or rather its lack, and poor disease control, which is particularly dangerous in patients treated for glaucoma or after ophthalmic surgery $[27,28]$. It has been proven that the smaller the number of drugs and the easier the dosing schedule, the greater patient adherence to treatment [29]. Satisfactory patient adherence cannot, however, be a priority in choosing a therapy. Medical considerations are of paramount importance. The ideal situation is one in which the patient can be offered a treatment that is consistent with the currently available medical knowledge, and at the same time, convenient to use.

\section{NEW THERAPEUTIC OPTION — LEVOFLOXACIN/DEXAMETHASONE}

The combination of levofloxacin and dexamethasone in one product, recently available in Poland, meets the needs of patients and doctors. One container contains substances with proven effectiveness: the recommended antibiotic - levofloxacin and the steroid - dexamethasone. It is an ideal solution in postoperative prophylaxis of endophthalmitis, and when used for seven days after ophthalmic surgery, it prevents the development of antibiotic resistance.

The randomized iPERME study [30] assessed the ability of a fixed combination of levofloxacin and dexamethasone vs. its single components to penetrate into the aqueous humor in patients undergoing cataract surgery. Drugs were administered twice: 90 and 60 minutes prior to paracentesis. The study was completed by 125 patients. The concentration of levofloxacin in the anterior chamber in the group that received the combination of levofloxacin and dexamethasone, compared with the group treated with levofloxacin alone, was not statistically significantly different and for both substances exceeded the MIC for the majority of gram-positive and gram-negative bacteria responsible for the development of postoperative inflammation of the eyeball. In contrast, the concentration of dexamethasone was slightly lower in the group that received the combination drug, compared to the reference group treated with dexamethasone alone, but within the range of pharmacodynamic activity. The results confirm that the fixed combination of levofloxacin and dexamethasone effectively penetrate the corneal epithelium and can achieve the desired pharmacological concentration after administration in the form of eye drops in patients undergoing cataract surgery [30].

Another large study evaluating the combination of levofloxacin and dexamethasone is LEADER 7 [31]. This is a phase III, randomized, international, multicenter, blinded trial comparing a treatment regimen of levofloxacin plus dexamethasone for seven days vs. the commonly used combination of tobramycin and dexamethasone for 14 days. This project assessed treatment efficacy, safety, and tolerability, and patient adherence. The study included a large group of 808 patients. There was no statistically significant advantage of the combination of tobramycin and dexamethasone used for 14 days over the new fixed combination of levofloxacin with dexamethasone used for seven days in the control of anterior chamber inflammation and conjunctival hyperemia after cataract surgery. There were no cases of endophthalmitis. This is the first study to describe a rational therapeutic strategy after cataract surgery and to demonstrate the correct use of antibiotics after surgery, including:

- short-term therapy (maximum one week); 
- administration of a broad-spectrum antibiotic - levofloxacin, active against microorganisms most often responsible for eye infections.

The study also re-assessed the need for steroid treatment after cataract surgery, showing that:

- short-term steroid therapy (one week) may be enough to control the inflammation in the anterior chamber and conjunctival hyperemia;

- gradual withdrawal may not be necessary.

The results of the LEADER 7 study suggest that an ophthalmic formulation containing a combination of levofloxacin and dexamethasone could be a reasonable addition to the current arsenal of medications available to treat/prevent inflammation and prevent infection following cataract surgery. It also provides an opportunity to review the treatment with topical glucocorticosteroids after ophthalmic surgery.

\section{SUMIMARY}

The Association of Polish Ophthalmology Surgeons (SCOP) has updated the guidelines for cataract surgery, indicating that although prospective studies (including the 2007 ESCRS trial [4]) did not confirm the efficacy of topical antibiotics in the postoperative period, in cases of uncertainty - whether the surgical incisions are tightly closed or when the patient is at risk of nosocomial infection - the use of antibiotics may be appropriate. At the same time, the SCOP recommends the administration of broad-spectrum fluoroquinolones. Drugs should be used intensively (in the maximum allowable dose) and briefly (5-7 days). In order to prevent the development of antibiotic resistance, the antibiotic should not be discontinued gradually (this also applies to fixed combinations). In all cases, the SCOP recommends administering the topical steroid for 21 days in tapering doses to minimize inflammation. In addition, the SCOP considered a preferred therapeutic option to use a fixed combination of levofloxacin and dexamethasone in the form of drops administered four times a day for seven days and then to continue only a topical glucocorticosteroid. In all people at increased risk of developing macular cystic edema (people with diabetes, patients with a history of uveitis, complicated cataract surgery, patients using prostaglandins for perioperative glaucoma, etc.), it is advisable to use a steroid in combination with an NSAID in accordance with SPC.

\section{REFERENCES}

1. Antimicrobial resistance. https://www.who.int/news-room/factsheets/detail/antimicrobial-resistance (26 May 2021).

2. D'Costa VM, King CE, Kalan L, et al. Antibiotic resistance is ancient. Nature. 2011; 477(7365): 457-461, doi: 10.1038/nature10388., indexed in Pubmed: 21881561.

3. Cassini A, Högberg L, Plachouras D, et al. Attributable deaths and disability-adjusted life-years caused by infections with antibioticresistant bacteria in the EU and the European Economic Area in 2015: a population-level modelling analysis. Lancet Infec Dis. 2019; 19(1): 56-66, doi: 10.1016/s1473-3099(18)30605-4, indexed in Pubmed: 30409683.

4. Barry P, Cordovés L, Gardner S. ESCRS Guidelines for Prevention and Treatment of Endophthalmitis Following Cataract Surgery: Prevention and Treatment Endophthalmitis. European Society for Cataract \& Refractive Surgeons, Dublin 2013.

5. MacGowan A, Macnaughton E. Antibiotic resistance. Medicine. 2017; 45(10): 622-628, doi: 10.1016/j.mpmed.2017.07.006

6. Vester B, Douthwaite $S$. Macrolide resistance conferred by base substitutions in 23S rRNA. Antimicrob Agents Chemother. 2001; 45(1): 1-12, doi: 10.1128/AAC.45.1.1-12.2001, indexed in Pubmed: 11120937.

7. O'Neill J. Tackling Drug-Resistant Infections Globally: Final Report And Recommendations The Review On Antimicrobial Resistance. https:// amr-review.org/sites/default/files/160518_Final\%20paper_with\%20 cover.pdf.

8. Ventola CL. The antibiotic resistance crisis: part 1: causes and threats. PT. 2015; 40(4): 227-283, indexed in Pubmed: 25859123.

9. Jackson TL, Johnston RL, Donachie PHJ, et al. The Royal College of Ophthalmologists' National Ophthalmology Database Study of Vitreoretinal Surgery: Report 6, Diabetic Vitrectomy. JAMA Ophthalmol. 2016; 134(1): 79-85; quiz 120, doi: 10.1001/jamaophthalmol.2015.4587, indexed in Pubmed: 26584210.

10. Endophthalmitis Study Group, European Society of Cataract \& Refractive Surgeons. Prophylaxis of postoperative endophthalmitis following cataract surgery: results of the ESCRS multicenter study and identification of risk factors. J Cataract Refract Surg. 2007; 33(6): 978-988, doi: 10.1016/j.jcrs.2007.02.032, indexed in Pubmed: 17531690.

11. Postępowanie okołooperacyjne w chirurgii zaćmy Wytyczne Polskiego Towarzystwa Okulistycznego. Stan na dzień 10 czerwca 2014. https:// adst.mp.pl/s/www/okulistyka/wytyczne_PTO_chirurgia_zacmy_2014. pdf.

12. Lindstrom RL, Galloway MS, Grzybowski $A$, et al. Dropless Cataract Surgery: An Overview. Curr Pharm Des. 2017; 23(4): 558-564, doi: 10 .2174/1381612822666161129150628, indexed in Pubmed: 27897120.

13. Chang DF, Braga-Mele R, Henderson BAn, et al. ASCRS Cataract Clinical Committee. Antibiotic prophylaxis of postoperative endophthalmitis after cataract surgery: Results of the 2014 ASCRS member survey. J Cataract Refract Surg. 2015; 41(6): 1300-1305, doi: 10.1016/j. jcrs.2015.01.014, indexed in Pubmed: 26189384.

14. Hecht I, Karesvuo P, Achiron A, et al. Anti-inflammatory Medication After Cataract Surgery and Posterior Capsular Opacification. Am J Ophthalmol. 2020; 215: 104-111, doi: 10.1016/j.ajo.2020.02.007, indexed in Pubmed: 32061757.

15. Krupin T, Mandell Al, Podos SM, et al. Topical corticosteroid therapy and pituitary-adrenal function. Arch Ophthalmol. 1976; 94(6): 919-920, doi: 10.1001/archopht.1976.03910030459003, indexed in Pubmed: 938282

16. Singh R, Alpern L, Jaffe GJ, et al. Evaluation of nepafenac in prevention of macular edema following cataract surgery in patients with diabetic retinopathy. Clin Ophthalmol. 2012; 6: 1259-1269, doi: 10.2147/0PTH. S31902, indexed in Pubmed: 22927737.

17. Hooper DC. Mechanisms of action and resistance of older and newer fluoroquinolones. Clin Infect Dis. 2000; 31 Suppl 2: S24-S28, doi: 10.1086/314056, indexed in Pubmed: 10984324

18. King DE, Malone R, Lilley, SH. New Classification and Update on the Quinolone Antibiotics. Am Fam Physician. 2000; 61(9): 2741-2748, indexed in Pubmed: 10821154.

19. Liu HH. Safety profile of the fluoroquinolones: focus on levofloxacin. Drug Saf. 2010; 33(5): 353-369, doi: 10.2165/11536360-00000000000000, indexed in Pubmed: 20397737. 
20. Schwab I, Friedlaender M, McCulley J, et al. A phase III clinical trial of $0.5 \%$ levofloxacin ophthalmic solution versus $0.3 \%$ ofloxacin ophthalmic solution for the treatment of bacterial conjunctivitis. Ophthalmology. 2003; 110(3): 457-465, doi: 10.1016/s0161-6420(02)01894-8, indexed in Pubmed: 12623805.

21. Ross D, Elkinton S, Riley C. Physicochemical properties of the fluoroquinolone antimicrobials. III. 1-0ctanol/water partition coefficients and their relationships to structure. Int J Pharm. 1992; 88(1-3): 379-389, doi: 10.1016/0378-5173(92)90336-z.

22. Sakamoto $H$, Sakamoto $M$, Hata $Y$, et al. Aqueous and vitreous penetration of levofloxacin after topical and/or oral administration. Eur $\mathrm{J}$ Ophthalmol. 2007; 17(3): 372-376, doi: 10.1177/112067210701700 316, indexed in Pubmed: 17534819.

23. Osato $M$, Jensen $H$, Trousdale $M$, et al. The Comparative In Vitro Activity of Ofloxacin and Selected Ophthalmic Antimicrobial Agents Against Ocular Bacterial Isolates. Am J Ophthalmol. 1989; 108(4): 380-386, doi: 10.1016/s0002-9394(14)73305-7.

24. Raizman M, Rubin J, Graves A, et al. Tear concentrations of levofloxacin following topical administration of a single dose of $0.5 \%$ levofloxacin ophthalmic solution in healthy volunteers. Clin Ther. 2002; 24(9): 1439-1450, doi: 10.1016/s0149-2918(02)80047-9, indexed in Pubmed: 12380635.

25. Keating GM. Moxifloxacin $0.5 \%$ ophthalmic solution: in bacterial conjunctivitis. Drugs. 2011; 71(1): 89-99, doi: 10.2165/11205840000000000-00000, indexed in Pubmed: 21175242.
26. Winfield AJ, Jessiman D, Williams $A$, et al. A study of the causes of non-compliance by patients prescribed eyedrops. $\mathrm{Br} \mathrm{J}$ Ophthalmol. 1990; 74(8): 477-480, doi: 10.1136/bjo.74.8.477, indexed in Pubmed: 2390523.

27. Kholdebarin R, Campbell R, Jin YP, et al. Multicenter study of compliance and drop administration in glaucoma. Can $\mathrm{J}$ Ophthalmol. 2008; 43(4): 454-461, doi: 10.3129/i08-076, indexed in Pubmed: 18711461.

28. An JA, Kasner 0, Samek DA, et al. Evaluation of eyedrop administration by inexperienced patients after cataract surgery. J Cataract Refract Surg. 2014; 40(11): 1857-1861, doi: 10.1016/j.jcrs.2014.02.037, indexed in Pubmed: 25248295.

29. Considerations in glaucoma therapy: fixed combinations versus their component medications. Clin Ophthalmol. 2009: 1, doi: 10.2147/opth. s6645, indexed in Pubmed: 20169043.

30. Figus M, Posarelli C, Romano D, et al. Aqueous humour concentrations after topical apPlication of combinEd levofloxacin-dexamethasone eye dRops and of its single components: a randoMised, assEssorblinded, parallel-group study in patients undergoing cataract surgery: the iPERME study. Eur J Clin Pharmacol. 2020; 76(7): 929-937, doi: 10.1007/s00228-020-02863-7, indexed in Pubmed: 32285142.

31. Levo-Dexa vs. Tobra+Dexa for Prevention and Treatment of Inflammation and Prevention of Infection in Cataract Surgery. https:// clinicaltrials.gov/ct2/show/NCT03739528 (25 June 2021). 